\title{
AVALIAÇÃO POLISSONOGRÁFICA DE PACIENTES AMBULATORIAIS PORTADORES DE TRANSTORNOS DO HUMOR
}

\author{
Mônica M. Souza', Damácio Ramón Kaimén Maciel², Rubens Reimão
}

\begin{abstract}
RESUMO - A avaliação do sono e os achados polissonográficos de 60 pacientes portadores de transtorno do humor, acompanhados em ambulatório, com média de idade de $36,1 \pm 11,3$ anos são apresentados ( $12 \mathrm{M}$, $48 \mathrm{~F}$ ). Os pacientes foram submetidos a entrevista clínica, questionário do sono e a duas noites de polissonografia, sendo a segunda noite utilizada para dados estatísticos. A distimia foi o diagnóstico mais comum em 25 casos. (41,6\%). Para o delineamento do estudo utilizou-se análise descritiva. As avaliações estatísticas empregadas foram o teste exato de Fisher e o teste de Kruskal-Wallis. Houve tendência para redução da continuidade do sono e aumento da densidade REM, em níveis estatisticamente não significantes. Ocorreu redução significante do sono profundo (estágio 4) diretamente relacionada com a maior severidade da depressão $(p=0,0253)$.
\end{abstract}

PALAVRAS-CHAVE: sono, distúrbios do sono, polissonografia, transtornos do humor, insônia.

\begin{abstract}
Polysomnographic evaluation of clinical patients suffering from mood disturbance
ABSTRACT - The sleep patterns of sixty patients suffering from mood disturbance, were studied, with mean age of $36.1 \pm 11.3$ years ( 12 males, 48 females). These patients were submitted to two nights of nocturnal polysomnographic evaluation; the second night acquired data was employed in the statistics. The following parameters were analyzed: parameters of sleep continuity, parameters of sleep architecture, REM and non-REM sleep parameters. The patients were analyzed in accordance to depression severity, presence of sleep disorders and relevant mood disturbances. The most common diagnosis was dysthymia in 25 cases (41.6\%). Descriptive analysis was used to delineate the study. The statistics evaluation used the Fisher exact test and the Kruskal-Wallis statistics heterogeneity test. There was reduction in sleep continuity and increase in REM density, both not statistic significant. Stage 4 sleep showed significant reduction in severely depressed patients $(p=0.0253)$.
\end{abstract}

KEY WORDS: sleep, sleep disorders, polysomnography, mood disorders, insomnia.

Os distúrbios do sono são manifestações clínicas da depressão e cerca de $50 \%$ a $95 \%$ dos pacientes portadores de transtornos do humor apresentam queixas de sono fragmentado e superficial ${ }^{1,2}$. A insônia do tipo terminal é o relato mais frequente e a hipersônia o menos comum ${ }^{1,2}$.

Os estudos de polissonografia em depressão focalizam principalmente a avaliação dos parâmetros da continuidade do sono, do sono REM e do sono nãoREM (NREM) ${ }^{3}$. Nas duas últimas décadas diversos autores verificaram inicialmente a característica polissonográfica da latência REM reduzida em quadros depressivos, sendo considerada por muitos como um marcador biológico da depressão $0^{4-6}$. As pesquisas mais recentes e de meta-análise têm corroborado e ampliado a complexidade das características polissonográficas associadas aos diversos transtornos do humor ${ }^{5,7-12}$.

Os transtornos do humor são sub-classificados em transtorno unipolar ou bipolar. $\mathrm{O}$ transtorno afetivo unipolar subdivide-se em episódio depressivo (leve, moderado ou grave), transtorno depressivo recorrente (leve, moderado ou grave), com ou sem sintomas psicóticos, e transtorno persistente do humor (distimia e ciclotimia) ${ }^{1,4,13}$.

No transtorno bipolar, os achados do sono são caracterizados de acordo com a fase da doença. Durante a fase depressiva, os pacientes têm sonolência

Universidade Estadual de Londrina (UEL), Londrina PR, Brasil; Divisão de Clínica Neurológica do Hospital das Clínicas da Faculdade de Medicina da Universidade de São Paulo (FMUSP), São Paulo SP, Brasil. 'Neurologista Clínica, Professora Auxiliar de Neurologia da UEL, Mestre em Medicina Interna; ${ }^{2}$ Professor Assistente de Neurologia da UEL; ${ }^{3}$ Professor Livre-Docente da Divisão de Clínica Neurológica do Hospital das Clínicas da FMUSP.

Recebido 30 Outubro 2002, recebido na forma final 10 Janeiro 2003. Aceito 20 Janeiro 2003. 
diurna excessiva e a duração do sono noturno está aumentada, com padrão característico de latência REM curta. Apesar da duração aumentada do sono total, os pacientes continuam a se queixar de sonolência diurna. Durante a fase maníaca, o sono é abreviado com uma redução drástica nas 24 horas'

Indivíduos que são cronicamente depressivos, por ao menos dois anos, mas que não completam os critérios para depressão maior por determinado tempo, são classificados como distímicos e constituem uma grande população de pacientes ambulatoriais ${ }^{13}$. A distimia apresenta achados polissonográficos variáveis, podendo ser normais ou alterados, a depender dos sintomas depressivos no momento da entrevista clínica e da realização do registro polissonográfico. As alterações consistem em latência REM curta ${ }^{4,13,14}$, comprometimento do sono profundo NREM com redução dos estágios 3 e 4, aumento da duração do primeiro período REM e despertares aumentados. A densidade REM pode estar aumentada. Os distímicos não se configuram como um grupo depressivo homogêneo ${ }^{4}$.

As co-morbidades como ansiedade, doença do pânico e traço obsessivo compulsivo são frequentes. Na ansiedade é comum a insônia inicial e a de manutenção. Na doença do pânico os ataques podem despertar o paciente. A polissonografia demons- tra a latência para iniciar o sono longa, interrupções por despertares longos, aumento do estágio 1 do sono, diminuição do sono profundo (estágios 3 e 4) e do sono REM. A latência REM está normal15-18.

Pacientes com depressão secundária a outros transtornos médicos ou psiquiátricos podem apresentar despertar matutino precoce e latência REM diminuída, mas são menos pronunciados do que em pacientes com o distúrbio primário ${ }^{19,20}$.

O objetivo do presente estudo é avaliar os distúrbios do sono encontrados em uma amostra de pacientes com transtornos do humor, bem como descrever suas principais características polissonográficas. Além disto, visa determinar as relações entre as variáveis polissonográficas (parâmetros de sono REM, NREM e medidas de continuidade de sono) e o grau de severidade da depressão.

\section{MÉTODO}

A amostra consistiu de 60 pacientes provenientes do ambulatório de Clínica Médica do Hospital Universitário Regional do Norte do Paraná encaminhados consecutivamente para avaliação neuropsiquiátrica, portadores de transtorno depressivo único ou misto, livres de drogas psicoativas e em particular de drogas hipnótico-sedativas por ao menos duas semanas anteriores ao estudo. Os pacientes foram submetidos a entrevista clínica com estabelecimento de critérios diagnósticos para transtornos do hu-

Tabela 1. Amostra de 60 pacientes com transtorno do humor. Resultados das escalas de Beck e Hamilton para depressão e variáveis analisadas na polissonografia com parâmetros do sono REM, parâmetros do sono não-REM e parâmetros da continuidade do sono.

\begin{tabular}{lccccc}
\hline Variáveis & Min & Máx & Mediana & Média & D. Padrão \\
\hline Idade & 16 & 59 & 36 & 36,1 & 11,35 \\
Latência REM (min) & 1 & 235 & 67 & 75,3 & 48,21 \\
Duração 1o REM (min) & 3 & 69 & 18 & 22,8 & 14,01 \\
\% Sono REM & 5,7 & 47,0 & 27,0 & 27,2 & 8,56 \\
\% Estágio 3 & 0 & 13,0 & 3,2 & 3,2 & 2,7 \\
\% Estágio 4 & 0 & 19,5 & 2,0 & 3,4 & 4,4 \\
\% Estágios 3 + 4 & 0 & 22,6 & 6,0 & 6,7 & 6,0 \\
Beck & 6 & 53 & 30 & 31,1 & 8,8 \\
Hamilton & 19 & 50 & 29,5 & 30,8 & 7,6 \\
Troca de estágios & 24 & 163 & 76,5 & 72,3 & 29,4 \\
Latência do sono (min) & 0 & 50 & 4,5 & 8,8 & 10,4 \\
No Total despertares & 4 & 58 & 19,5 & 21,2 & 10,7 \\
Eficiência do sono & 52,1 & 98,5 & 93,6 & 90,5 & 10,1 \\
T. total de vigília (min) & 6 & 192 & 24 & 35,4 & 36 \\
\hline & & & & & \\
\hline
\end{tabular}


Tabela 2. Comparação dos resultados das escalas de Beck e Hamilton para depressão e de variáveis polissonográficas entre os pacientes com depressão leve, moderada e grave.

\begin{tabular}{|c|c|c|c|c|c|c|c|}
\hline \multirow[b]{2}{*}{ Variáveis } & \multirow[b]{2}{*}{$P$} & \multicolumn{2}{|c|}{$\begin{array}{l}\text { Leve } \\
(n=9)\end{array}$} & \multicolumn{2}{|c|}{$\begin{array}{l}\text { Moderada } \\
\qquad(n=26)\end{array}$} & \multicolumn{2}{|c|}{$\begin{array}{c}\text { Grave } \\
(n=25)\end{array}$} \\
\hline & & Média & DP & Média & DP & Média & DP \\
\hline Idade & 0,7725 & 33,7 & 12,1 & 36,2 & 12,8 & 36,9 & 9,6 \\
\hline Latência REM (min) & 0,3097 & 62,1 & 28,2 & 86,0 & 57,9 & 69,0 & 41,6 \\
\hline Duração 1 REM (min) & 0,5246 & 27,3 & 19,4 & 21,1 & 10,6 & 22,9 & 15,2 \\
\hline$\%$ Sono REM & 0,1785 & 31,9 & 9,9 & 26,1 & 8,9 & 26,3 & 7,1 \\
\hline \% Estágio 3 & 0,7534 & 2,8 & 1,3 & 3,5 & 2,9 & 3,1 & 2,9 \\
\hline \% Estágio 4 & 0,0253 & 6,7 & 6,5 & 3,6 & 4,0 & 2,1 & 3,5 \\
\hline \% Estágios 3 + 4 & 0,1610 & 9,5 & 7,2 & 7,2 & 5,7 & 5,2 & 5,6 \\
\hline BECK & 0,0001 & 18,9 & 5,6 & 27,8 & 2,7 & 38,9 & 6,4 \\
\hline HAMILTON & 0,0001 & 21,4 & 1,5 & 27,9 & 4,3 & 37,2 & 6,2 \\
\hline Troca de estágios & 0,8671 & 71,6 & 21,1 & 70,3 & 29,7 & 74,7 & 32,3 \\
\hline Latência do sono (min) & 0,7416 & 7,0 & 6,7 & 7,0 & 5,1 & 11,3 & 14,7 \\
\hline N. Total despertares & 0,4781 & 19,4 & 6,4 & 20,0 & 10,5 & 23,2 & 12,0 \\
\hline Eficiência do sono & 0,8075 & 93,5 & 4,1 & 90,8 & 9,2 & 89,0 & 12,3 \\
\hline T. total de vigília (min) & 0,7579 & 25,0 & 16,4 & 33,9 & 32,9 & 40,7 & 44,8 \\
\hline
\end{tabular}

mor e de ansiedade de acordo com o Manual de Diagnóstico e Estatística DSM IV ${ }^{21}$ e a Classificação Internacional das Doenças CID 1022. Além disto, foram aplicadas as escalas de Beck $^{23}$ e de Hamilton ${ }^{24}$ para depressão. Foram registrados os antecedentes pessoais e familiares, fatores de estresse e questionário do sono. A respeito do sono, inquiriu-se sobre o tempo de adormecimento, a existência de insônia ou hipersônia, os despertares noturnos precoces, qualidade e quantidade dos mesmos. Foi indagada a existência de outros fenômenos do sono como mioclonias do sono ou dificuldade respiratória. Esta pesquisa foi aprovada pela Comissão de Ética (Presidente Dr. José Eduardo de Siqueira) e os pacientes preencheram o respectivo Termo de Consentimento Livre e Esclarecido.

Cada paciente foi estudado por meio da polissonografia em duas noites de sono, utilizando-se para fins estatísticos a segunda noite, com registro polissonográfico de 6 horas. A leitura dos registros polissonográficos obedeceu os critérios padronizados por Rechtschaffen e Kales ${ }^{25}$ e recomendados pela Associação Americana de Distúrbios do Sono ${ }^{18}$.

As variáveis foram as seguintes: $A$ ) índices de continuidade do sono (tempo total de registro, tempo total de sono, eficiência do sono, despertar intermitente, despertar matutino precoce); B) índices de arquitetura do sono (percentagem dos estágios 1, 2, 3 e 4 e do sono REM); C) índices do sono REM (latência REM, percentagem do sono REM, densidade REM [movimentos oculares no determi-
Tabela 3. Principais queixas do sono na amostra total de 60 pacientes com transtornos do humor.

\begin{tabular}{lc}
\hline Queixas do sono & $\mathrm{N}(\%)$ \\
\hline Insônia & $50(83,3 \%)$ \\
Hipersônia & $09(15,0 \%)$ \\
Sem queixas & $01(1,67 \%)$ \\
Total & $60(100 \%)$ \\
\hline
\end{tabular}

nado tempo de sono REM], atividade REM média [medida da atividade dos movimentos oculares rápidos REM dividida pelo tempo total de sono]). A densidade REM foi realizada por meio da contagem visual dos movimentos oculares rápidos durante todos os períodos REM e selecionados a partir da amplitude de $25 \mu \mathrm{V}$.

Foram excluídos quatro pacientes: um idoso com disfunção cognitiva, uma paciente que mudou-se para longe do Paraná, uma paciente que engravidou e um paciente com tentativa de suicídio.

Para o delineamento estatístico do estudo utilizou-se inicialmente um inquérito descritivo, tendo em vista os diversos subtipos de transtornos depressivos e ansiosos, tratando-se de uma população não-homogênea.

As variáveis qualitativas (sexo e idade) foram submeti- 
das ao teste exato de Fisher. As variáveis quantitativas (parâmetros de sono REM, parâmetros de sono NREM e parâmetros de continuidade de sono) foram avaliadas por meio da estatística descritiva (média artimética, desvio padrão, valor mínimo, valor máximo e medina).

Frequência foi utilizada para quantificar as variáveis sexo e severidade da depressão. O valor mínimo, valor máximo, mediana, média e o desvio padrão foram utilizados para descrever as variáveis idade, tempo REM em minutos, duração REM em minutos, percentagem REM, percentagem do sono em estágios 3 e 4, escala de Beck e escala de Hamilton, latência do sono em minutos, número de trocas de estágios, número total de despertares, eficiência do sono e tempo total de vigília em minutos.

Para comparar os grupos de severidade leve, moderado e grave em relação às variáveis quantitativas utilizouse: 1 - análise de variância e teste de Tukey para comparação das médias, duas a duas, quando o teste de Barlett indicava homogeneidade das variâncias e o teste de Shapiro-Wilk indicava a normalidade dos dados; 2 - teste de Kruskal-Wallis e teste de comparações múltiplas das médias das ordens quando o teste de Barlett indicava a heterogeneidade das variâncias e/ou teste de Shapiro-Wilk indicava a não normalidade dos dados.

Adotou-se o nível de significância de $5 \%(\alpha=0,05)$ em todos os testes.

\section{RESULTADOS}

A amostra estudada constou de 60 pacientes, com média de idade de $36,1 \pm 11,3$ anos ( $12 \mathrm{M}, 48$ $\mathrm{F}$ ). As informações referentes às variáveis qualitativas e quantitativas encontram-se na Tabela 1.

Com relação ao estudo da densidade REM, a percentagem média foi de $22,4 \pm 10,26 \%$. Para o grupo de depressivos leve, moderado e grave, os valores respectivos foram os seguintes: $21,6 \pm 10,5 \%$, $21,8 \pm 10,5 \%$ e $23,4 \pm 10,3 \%$.

Na Tabela 2 consta o estudo das variáveis qualitativas e quantitativas entre os diversos grupos de depressão. Na Tabela 3, observa-se a maior prevalência de insônia e menor de hipersônia na amostra estudada.

No teste de comparações múltiplas para as médias das severidades a percentagem do estágio 4 mostrou-se reduzida no grupo depressivo severo $(p=0,0253)$.

O diagnóstico mais frequente de acordo com a CID $10^{22}$ foi distimia isoladamente ou em associação em 25 casos $(41,67 \%)$. Os outros diagnósticos foram: transtorno do pânico; transtorno obsessivo compulsivo; anorexia nervosa; transtorno de personalidade emocionalmente instável tipo impulsivo; transtorno mental e de comportamento decorrente do uso de álcool; e transtorno depressivo orgânico (hipotireoidismo). Estes pacientes foram classificados como portadores de depressão forma secundária (16 pacientes da amostra). Apenas um paciente era portador de depressão psicótica.

Com o objetivo de homogeneizar a amostra 9 pacientes ( 1 paciente com sono REM de $1 \%$, 4 pacientes do grupo depressivo moderado com latência REM acima de 120 minutos e 4 pacientes do grupo depressivo severo) foram excluídos para nova análise estatística. Demonstrou-se nesta análise a redução da latência REM, sem significância estatística.

\section{DISCUSSÃO}

Considerando o número de pacientes nessa amostra em estudo e o tipo de delineamento utilizado não foi possível inferir comparações estatísticas dos achados polissonográficos entre os subgrupos de transtornos do humor e seus subtipos. Em relação à origem dos pacientes, a amostra analisada tem base no estudo de pacientes provenientes de atendimento primário e ambulatorial onde a predominância diagnóstica é de distimia que apresenta achados polimórficos nos resultados polissonográficos conforme relatado por Akiskal et al. ${ }^{4}$ em 1997. Estes podem variar ${ }^{4,10,13}$ desde a normalidade até as descrições típicas com redução dos estágios profundos de sono e aumento da densidade REM se assemelhando aos resultados encontrados na presente casuística.

A latência REM média na presente casuística situou-se em torno de $75 \mathrm{~min}$, valor este diminuído em relação aos índices normais. Estudos fundamentais ${ }^{26}$ colocam os valores de latência REM na depressão primária como em média de 51,3 min; na depressão secundária como sendo de 75,9 min; e em outros transtornos psíquicos com resultados de média de $76,5 \%$. Na presente amostra não foi possível estabelecer correlação significante entre os subgrupos de severidade e achados de latência REM reduzida, correlação esta que foi verificada por Feinberg et al. ${ }^{27}$. O estudo de meta-análise realizado por Knowles et al. ${ }^{5}$ avaliando 27 publicações comprovou a relevância da redução da latência REM em grupos de pacientes depressivos, estando portanto de acordo com os achados da presente casuística.

$\mathrm{Na}$ amostra em estudo foi possível observar, em 5 casos, a associação entre depressão e doença do pânico. Diversas publicações ${ }^{6,10}$ têm evidenciado o perfil de sono dos pacientes portadores dessas duas co-morbidades ocupando uma posição intermediária entre as descrições polissonográficas clássicas do transtorno do pânico e do transtorno depressivo. 
As descrições típicas da literatura apontam que na depressão o primeiro período REM pode ser longo e mesmo predominar no primeiro terço da noi$\mathrm{te}^{28}$. Esta característica polissonográfica foi comprovada em estudos recentes e com meta-análise ${ }^{10}$. $\mathrm{Na}$ presente casuística, a duração do primeiro período REM está aumentada concordando com estes dados da literatura.

No presente estudo, a densidade REM média está aumentada. Diversos estudos recentes e com metaanálise comprovaram o aumento da densidade REM como um dos principais índices confiáveis e característicos dos pacientes com depressão e evidenciaram que este aumento tende a ser correlacionado com a severidade da depressão $0^{5,9,10}$. Em publicação recente, de 2002, Modell et al. ${ }^{7}$ verificaram que a densidade REM foi a principal medida polissonográfica consistente em estudo prospectivo longitudinal de um grupo de pacientes com doenças afetivas comparadas aos controles normais.

A percentagem de sono REM encontrada na presente casuística situa-se próxima aos valores normais. Diversos autores relatam o aumento de percentagem do sono REM em depressivos ${ }^{29}$ embora esta não seja uma característica uniforme na literatura ${ }^{9,10}$.

Em relação ao sono profundo (estágio 4) na presente casuística os valores estão diminuídos e houve maior redução no estágio 4 nos depressivos graves ao serem comparados com os depressivos leves. Este dado confirma os achados da literatura recente e com meta-análise que mostra a redução do sono profundo (estágios 3 e 4) como uma das principais características dos quadros depressivos ${ }^{5,8-11}$ embora possa ser detectada em outros distúrbios do sono que fragmentem a continuidade do sono.

A latência do sono na presente casuística encontrou-se aumentada em casos nos quais a depressão estava associada aos transtornos de ansiedade. $\mathrm{O}$ aumento da latência do sono é característico dos quadros de ansiedade portanto este dado da presente casuística concorda com a literatura ${ }^{5,6,8,9,9,17,26,28-30}$.

\section{CONCLUSÃO}

Com base na presente casuística pode-se concluir que o estudo da polissonografia mostrou-se útil como instrumento de quantificação e qualificação das alterações do sono nos transtornos do humor. Houve tendência para redução da continuidade do sono e aumento da densidade REM, em níveis estatisticamente não significantes. Ocorreu redução do sono profundo (estágio 4) diretamente relacionada com a maior severidade da depressão $(p=0,025)$.
Agradecimentos - Agradecemos ao Grupo de Neuropsicoendocrinoimunologia da UEL na pessoa da Dra. Sandra Vargas Nunes, a análise estatística realizada pela Dra. Tieme Matsuo e o incentivo do amigo Dr. Fábio Nunes Trevisan.

\section{REFERÊNCIAS}

1. Kales A, Bixler EO, Vela-Bueno A, Cadieux RJ, Soldatos CR, Kales JD. Biopsychobehavioral correlates of insomnia. III. Polygraphic findings of sleep difficulty and their relationship to psychopathology. Int J Neurosci 1984;23:43-55.

2. Reynolds CF, Taska LS, Jarrett CB, Coble PA, Kupfer DJ. REM latency in depression: is there one best definition? Biol Psychiatry 1983;8:849-863.

3. Hayakawa T, Ohta T. Polysomnographic and other methods for assessment of sleep disorders. Nippon Rinsho 1998;56:354-360.

4. Akiskal HS, Judd LL, Gillin JC, Lemmi H. Subthreshold depressions clinical and polysomnographic validation of dysthymic, residual and masked forms. J Affect Disorder 1997;45:53-63.

5. Knowles JB, MacLean AW. Age-related changes in depressive and healthy subjects. A meta-analysis. Neuropsychopharmacology 1990;3:251-259.

6. Vgontzas NA, Kales A, Bixler EO, Manfredi RL, Vela-Bueno A Usefulness of polysomnographic studies in the differential diagnosis of insomnia. Int J Neurosci 1995;82:47-60.

7. Modell S, Ising M, Holboer F, Lauer CJ. The Munich Vulnerability Study on Affective Disorders: stability of polysomnographic findings over time. Biol Psychiatry 2002;52:430- 437.

8. Hudson JL, Pope HG, Sullivan LE, Watermaux CM, Keck PE, Broughton RJ. Good sleep, bad sleep: a meta-analysis of polysomnographic measures of insomnia, depression and narcolepsy. Biol Psychiatry 1992;32:958-975.

9. Jindal RD, Thase ME, Fasiczka AL, et al. Electroencephalographic sleep profiles in single-episode and recurrent unipolar forms of major depression: II. Comparison during remission. Biol Psychiatry 2002;51:230-236.

10. Riemann D, Berger M, Voderholzer U. Sleep and depression - results from psychobiological studies: an overview. Biol Psychol 2001;57:67-103.

11. Benca RM, Obermeyer WH, Thisted RA, Gillin JC. Sleep and psychiatric disorders. A meta-analysis. Arch Gen Psychiatry 1992;49:651-668.

12. Wichniak A, Riemann D, Klemen A, Voderholzer U, Jernajczy RW. Comparison between eye movement latency and REM sleep parameters in major depression. Eur Arch Psychiatry Clin Neurosci 2000;250:48-52.

13. Saletu-Zyhlarz GM, Abu-Bakr MH, Andersen P, et al. Insomnia related to dysthymia: polysomnographic and psychometric comparison with normal controls and acute therapeutic trials with trazodone. Neuropsychobiology 2001;44:139-149.

14. Akiskal HS, Lemmi H, Yerevanian B et al. The utility of REM latency test in psychiatric diagnosis. A study of 81 outpatients. Psychiatr Res 1982;7:101-110.

15. Aldrich MS. Polysomnographic assessment of insomnia. Sleep 1990;13:188-189.

16. Lamarche CE, Ogilvie RD. Electrophysiological changes during sleep onset period of psychophysiological insomniacs, psychiatric insomniacs, and normal sleepers. Sleep 1997;9:724-733.

17. Bourdet C, Goldenberg F. Insomnia in anxiety: sleep EEG changes. J Psychosom Res 1994;38(Suppl 1):93-104.

18. Practice parameters of the polysomnography in the evaluation of insomnia. Standards of Practice Committee of the American Sleep Disorders Association. Sleep 1995;18:55-57.

19. Clark CP, Gillin JC, Golshan S, et al. Polysomnography and depressive symptoms in primary alcoholics with and without a lifetime diagnosis of secondary depression in patients with primary major depression. J Affect Disord 1999;52:177-185.

20. Giles DE, Biggs MM, Roffwarg HP, Orsulak PJ, Rush AJ. Secondary depression: a comparison among subtypes. J Affect Disord 1987;12:251-258.

21. DSM-IV. Manual diagnóstico e estatístico de transtornos mentais. Porto Alegre, Artes Médicas, 1995.

22. Classificação dos transtornos mentais e do comportamento da CID-10. Organização Mundial da Saúde. Porto Alegre, Artes Médicas, 1993.

23. Beck AT, Ward CH, Mock J, Erbaugh J. An inventory for measuring depression. Arch Gen Psychiatry 1961;4:561-571.

24. Hamilton M. A rating scale for depression. J Neurol Neurosurg Psychiatr 1960;23:56-62.

25. Rechtschaffen A, Kales A. A manual of standardized terminology, techniques and scoring system for sleep stages of human subjects. Washington DC, USA, Government Printing Office, 1968.

26. Seifritz E. Contribution of sleep physiology to depressive pathophysiology. Neuropsychopharmacology 2001;25:85-88.

27. Feinberg M, Carroll BJ, Greden JF, Zis AP.Sleep EEG, depression rating scales and diagnosis. Biol Psychiatry 1982;17:1453-1462.

28. Idzikowski C. Insomnia and depression - sleep EEG changes. J Psychosom Res 1994;38(Suppl 1):27-40.

29. Rotenberg VS, Indursky P, Kayumov L, Sirota P, Melamed Y. The relationship between subjective sleep estimation and objective sleep variables in depressed patients. Int J Psychophysiol 2000;37:291-297.

30. Edinger JD, Hoelscher TJ, Webb MD, Marsh GR, Radtke RA, Rewin CW. Polysomnographic assessment of DIMS: empirical evaluation of its diagnostic value. Sleep 1989;12:315-322. 